EPJ Web of Conferences 49, 17014 (2013)

DOI: $10.1051 /$ epjconf/20134917014

(C) Owned by the authors, published by EDP Sciences, 2013

\title{
Top-pair production in hadron collisions at NNLL
}

\author{
M. Beneke ${ }^{1,2}$, P. Falgari ${ }^{3}$, S. Klein ${ }^{2}$, J. Piclum², C. Schwinn ${ }^{4}$, M. Ubiali ${ }^{2}$, and F. Yan ${ }^{2}$ \\ ${ }^{1}$ Physik Department T31, James-Franck-Straße, Technische Universität München, D-85748 Garching, Germany \\ ${ }^{2}$ Institut für Theoretische Teilchenphysik und Kosmologie, RWTH Aachen University, D-52056 Aachen, Germany \\ ${ }^{3}$ Institute for Theoretical Physics and Spinoza Institute, Utrecht University, 3508 TD Utrecht, The Netherlands \\ ${ }^{4}$ Albert-Ludwigs Universität Freiburg, Physikalisches Institut, D-79104 Freiburg, Germany
}

\begin{abstract}
We present results for the total top-pair production cross section at the Tevatron and the LHC. Our predictions supplement fixed-order results with resummation of soft logarithms and Coulomb singularities to next-to-next-to-leading logarithmic accuracy and include top-antitop bound-state effects. The effects of resummation, the dependence on the PDF set used, the residual sources of theoretical uncertainty and their implication for measurements of the top-quark mass are discussed.
\end{abstract}

\section{Introduction}

With hundreds of thousands of top quarks being produced yearly at the LHC, top-quark measurements are soon going to reach an unprecedented precision. In fact, the total top-pair production cross section has been measured by the two LHC collaborations with a total error of $\pm(4-6) \%[1,2]$, which is already comparable to, or below, the accuracy of analogous Tevatron results [3, 4]. With more statistics being collected at the LHC and a better understanding of systematic uncertainties, the error on the total $t \bar{t}$ production rate will be reduced even further. Measurements of the inclusive cross section provide an important test of the Standard Model (SM) and constrain newphysics effects. They can also be used to extract the topquark mass in a theoretically clean way and to constrain the gluon PDF at medium-large values of the Bjorken variable $x$. However, this is only possible if theoretical uncertainties are comparable to, or smaller than, the present experimental errors.

At fixed order in perturbation theory, the cross section has been known with next-to-leading (NLO) order accuracy for more than 20 years [5]. However, the theoretical uncertainty due to this result is about $\pm 10 \%$, and thus bigger than the experimental uncertainty at the Tevatron and the LHC. This makes it necessary to compute higher order corrections to improve the theoretical predictions. Recently, fixed-order calculations at the next-to-next-toleading order (NNLO) have been completed for the $q \bar{q}$ partonic production channel, which is relevant for $t \bar{t}$ phenomenology at the Tevatron, and for the $q g$ channel $[6,7]$. Only partial results exist for the $g g$ channel, which is the dominant one at the LHC.

Beyond fixed-order perturbation theory, theoretical predictions can be improved by summing certain sets of contributions to all orders. For the total cross section there are two kinds of contribution, which can be computed, and thus resummed, to all orders: threshold logarithms and Coulomb singularities. The former are due to the emission of soft gluons from the initial and the final state, while the latter arise from the non-relativistic interaction of the heavy-quark pair in the final state. Both corrections are enhanced near the partonic production threshold $\sqrt{\hat{s}}=2 m_{t}$, scaling respectively as $\left(\alpha_{s} \ln ^{2,1} \beta\right)^{n}$ and $\left(\alpha_{s} / \beta\right)^{n}$, with the velocity $\beta$ of the final top (antitop) defined in terms of the partonic centre-of-mass energy as $\beta=\sqrt{1-4 m_{t}^{2} / \hat{s}}$.

Results for the cross section including resummation of soft logarithms at next-to-leading logarithmic (NLL) accuracy have been available for more than 10 years $[8,9]$. Recent advances in the understanding of the infrared structure of massive QCD amplitudes [10,11] and the calculation of the relevant anomalous dimensions [12, 13], have made it possible to extend the resummation to nextto-next-to-leading logarithmic (NNLL) accuracy, and several groups have published results for the cross section at this order [14-17]. The factorization of soft and Coulomb effects has been proven in [18]. Based on this result, a combined resummation of soft and Coulomb corrections at NNLL accuracy has been presented in [19]. It is the only available prediction for the inclusive top-pair production cross section that resums both classes of corrections, including effects from $t \bar{t}$ bound states below threshold. Explicit results of the calculation of [19] are given in the following. All results have been computed with the userfriendly program TOPIXS [20], which implements the NNLL soft-Coulomb resummation as described in [19].

\section{The $t \bar{t}$ total cross section at NNLL}

In order to improve the behaviour at large $\beta$, the resummed cross section is matched to the fixed order one. In the case 
Table 1. Total $t \bar{t}$ cross section at NLO, NNLO and NNLL for Tevatron and LHC with $\sqrt{s}=7,8 \mathrm{TeV}$ and $m_{t}=173.3 \mathrm{GeV}$. The first set of errors refers to the theoretical uncertainty, the second to the $\mathrm{PDF}+\alpha_{s}$ uncertainty. All the numbers are in picobarns.

\begin{tabular}{lccc}
\hline$\sigma_{t \bar{t}}[\mathrm{pb}]$ & Tevatron & LHC $(7 \mathrm{TeV})$ & LHC $(8 \mathrm{TeV})$ \\
\hline NLO & $6.68_{-0.75-0.22}^{+0.36+0.23}$ & $158.1_{-21.2-6.2}^{+19.5+6.8}$ & $226.2_{-29.7-8.3}^{+27.8+9.2}$ \\
NNLO & $7.00_{-0.21+0.29}^{+0.21-0.25}$ & $160.9_{-11.1+7.6 .7}^{+11.1+2}$ & $229.8_{-16.7-9.7}^{+16.5+9.7}$ \\
NNLL & $7.15_{-0.20-0.25}^{+0.21+0.30}$ & $162.4_{-6.9-6.8}^{+6.7+7.3}$ & $231.8_{-9.9-9.1}^{+9.6+9.8}$ \\
\hline
\end{tabular}

of the $q \bar{q}$ channel the exact fixed-order NNLO cross section [6] is used, as detailed in Eq. (2.2) of [20]. This gives (almost) full NNLO+NNLL accuracy at Tevatron, where the $q \bar{q}$ production channel dominates the hadronic cross section. For the matching of the $g g$ channel, which is dominant at the LHC, TOPIXS uses the approximated NNLO result of [21], which contains all the threshold-enhanced terms at NNLO, but no constants at $O\left(\alpha_{s}^{4}\right)$.

In Table 1 we present results for the total top-pair cross section at NLO, NNLO and (matched) NNLL accuracy for Tevatron and LHC with $\sqrt{s}=7,8 \mathrm{TeV}$ and $m_{t}=173.3 \mathrm{GeV}^{1}$. The central value for both renormalization and factorization scale is set to $m_{t}$. For the convolution of the partonic cross sections with the parton luminosities we use the MSTW2008 PDF sets [22], using the NLO set for the NLO cross section and the NNLO set for NNLO and NNLL cross sections. The two sets of errors refer to the theoretical uncertainty of the approximation and to the combined PDF and $\alpha_{s}$ error obtained with the $68 \%$ confidence-level PDF set. The theoretical uncertainty is obtained from scale variation for the NLO result, from the sum of scale uncertainty and ambiguities related to unknown $O\left(\alpha_{s}^{4}\right)$ constant terms at NNLO and from the sum of scale, constant and resummation uncertainties for the resummed NNLL result [19]. Note that the error from the constant NNLO terms affects only the $g g$ channel, since the matching to the exact NNLO result for $q \bar{q}$ removes the uncertainty for this channel.

From Table 1 it can be seen that at Tevatron corrections beyond NLO are sizeable, corresponding to an upward shift of the cross section by $7 \%$. Of this, about $5 \%$ is accounted for by NNLO contributions, with higher-order terms from resummation contributing only $2 \%$ of the cross section. The situation is quite different at the LHC, where terms beyond NLO are only $3 \%$ of the NLO cross section, of which only about $1 \%$ originate from terms beyond $O\left(\alpha_{s}^{4}\right)$. Note that the positive contribution of higherorder terms is partly compensated at the hadronic level by a downward shift due to the switch from NLO to NNLO PDFs.

The addition of higher-order terms beyond NLO leads to a significant reduction of the theoretical uncertainty on the $t \bar{t}$ cross section. This is particularly true at the Tevatron, where the inclusion of the exact NNLO result for the dominant $q \bar{q}$ channel completely removes the uncertainty

\footnotetext{
${ }^{1}$ In Table 1 we use the same notation for Tevatron and LHC, though, strictly speaking, the LHC results are not exact at NNLO. The same is true for the matched NNLL cross section.
}

Tevatron

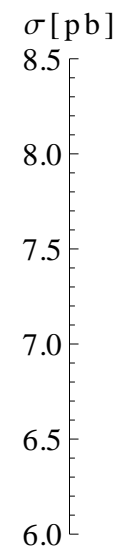

LHC (8 TeV)
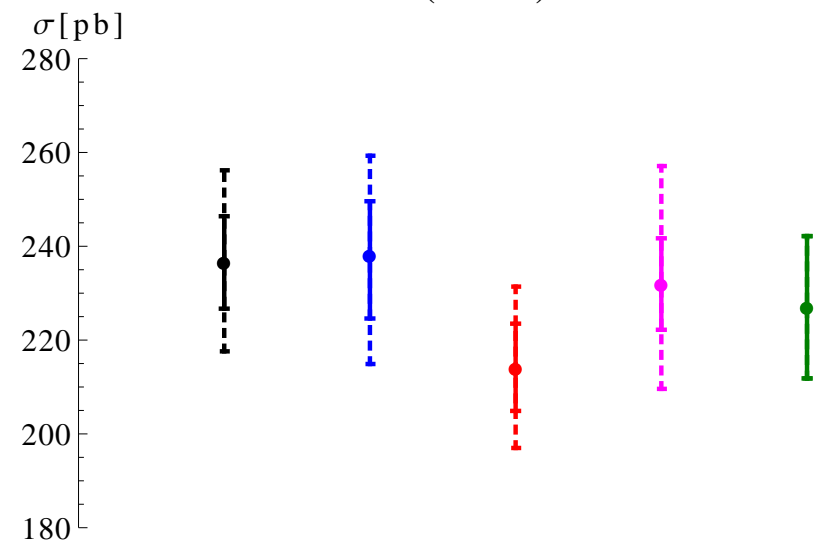

Figure 1. Comparison of the NNLL predictions obtained with different PDF sets and of recent experimental measurements of the total $t \bar{t}$ cross section, for Tevatron (upper plot) and LHC with $\sqrt{s}=8 \mathrm{TeV}$ (lower plot). The PDF sets are (from left to right): MSTW2008 (black), NNPDF2.1 (blue), ABM11 (red), and CT10 (magenta). The experimental results (green) are shown at the very right of the plots. The two error bars for the theoretical numbers represent total theoretical uncertainty (solid bar) and the $\mathrm{PDF}+\alpha_{s}$ uncertainty at $68 \%$ confidence level (dashed bar).

related to unknown $O\left(\alpha_{s}^{4}\right)$ terms. The residual theory error for the NNLL result is about $\pm 3 \%$, which is smaller than the error of the most recent experimental measurements. At the LHC the remaining theoretical uncertainty is slightly larger $( \pm 4 \%)$, due to the unknown NNLO constant contributions to the $g g$ production channel. Both at Tevatron and LHC the PDF $+\alpha_{s}$ error accounts for an additional $\pm(4-4.5) \%$ uncertainty.

Since the PDF $+\alpha_{s}$ error represents now the largest individual source of uncertainty, it is important to ascertain whether the error estimate provided by one of the many PDF parameterizations available is consistent with the spread of the central-value predictions obtained with the different sets. This is investigated in Figure 1, where the NNLL results for the total cross section obtained with the MSTW2008 [22], CT10 [23], NNPDF2.1 [24] and ABM11 [25] NNLO PDF sets are compared to each other and to the combined result of CDF and D0 [26] for the Tevatron and of CMS [27] for the LHC with $8 \mathrm{TeV}$ centre- 


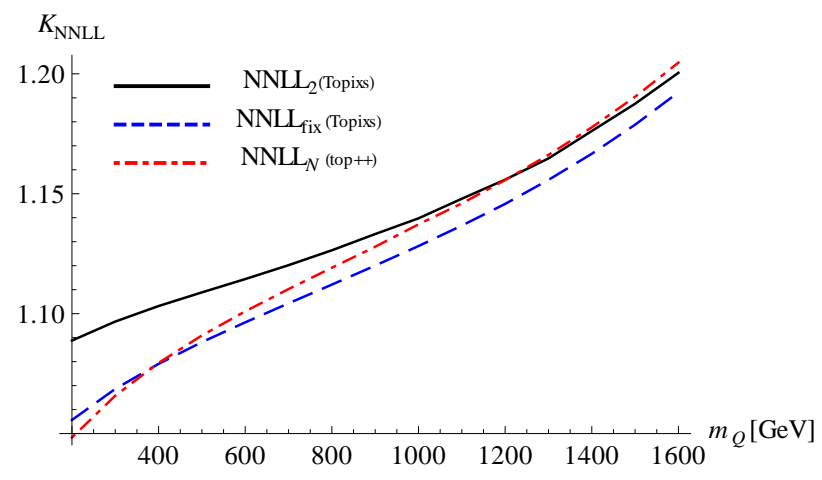

Figure 2. The ratio of the NNLL and NLO cross section is shown as a function of the quark mass at the LHC with $\sqrt{s}=8 \mathrm{TeV}$. The different lines correspond to our default implementation $\mathrm{NNLL}_{2}$ (black, solid), our implementation with fixed soft scale NNLL $_{\text {fix }}$ (red, dashed) and the Mellin-space result [17, 28] NNLL $_{N}$ (red, dot-dashed).

of-mass energy (note that the experimental results use the reference mass $m_{t}=172.5 \mathrm{GeV}$ ). To make the comparison of the different sets more transparent we use a common initial value of the strong coupling constant, $\alpha_{s}\left(M_{Z}\right)=0.118$. At the Tevatron, the agreement between different PDF sets is very good, and the central values for different PDF sets are compatible with the error estimate of the individual sets. Furthermore, the NNLL theoretical predictions are remarkably close to the experimental values provided by D0 and CDF. At the LHC, MSTW2008, CT10 and NNPDF2.1 still show a good agreement with each other and with the experimentally measured cross section. However, the result obtained with ABM11 differs significantly from the others, more than one would expect from the error estimate of the individual sets. This discrepancy can be traced to large differences in the gluon PDF between ABM11, which does not include Tevatron jet data in its fits, and the other three sets in the region of medium-large Bjorken variable $x$, which is the most relevant to $t \bar{t}$ production.

The difference observed at the LHC raises the question of whether measurements of the $t \bar{t}$ cross section can be used to constrain the gluon PDF at medium-large $x$. This was investigated in [20], using the reweighting procedure of the NNPDF collaboration to incorporate the information from recent $t \bar{t}$ measurements at the LHC and our NNLL prediction. It was found that the additional top-pair production input leads to a significant reduction of the uncertainties on the gluon parton luminosity. Also, in the case of the NNPDF2.1-DIS+DY sets, that do not include Tevatron jet data and whose gluon distribution is closer to the ABM11 one, the reweighting gives an upward shift of the gluon PDF that brings it close to the standard NNPDF2.1 distribution.

While the effect of resummation is small for $t \bar{t}$ production, it has been shown in [20] that NNLL corrections beyond NLO can amount to $15-20 \%$ for higher masses. This can be seen in Figure 2, where the ratio of the NNLL and NLO cross section is shown as a function of the quark

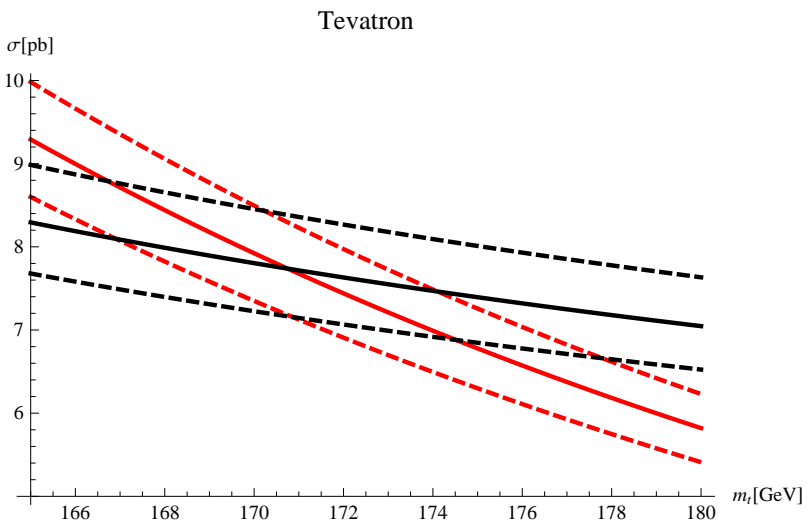

Figure 3. Mass dependence of the experimental $t \bar{t}$ cross section at Tevatron [3] (black) and of the NNLL resummed result provided by TOPIXS [20] (red). Solid lines represent the central values, while dashed lines give the total experimental and theoretical uncertainties.

mass, $m_{Q}$, for the LHC with $8 \mathrm{TeV}$ centre-of-mass energy. Thus, resummation becomes relevant in the context of searches for new SU(3)-triplet fermions, e.g. in fourth generation searches. Furthermore, the plot also shows that the agreement with the result of Ref. [17], where softgluon resummation is performed in Mellin space, becomes better as the mass increases. This is expected since the larger the mass is, the closer to threshold are the produced particles, and thus the smaller are the inherent ambiguities of the two methods.

\section{Top-mass extraction}

As pointed out in the introduction, measurements of the total $t \bar{t}$ cross section can be used to extract the top-quark mass from data. This was done, for example, in [29] using different higher-order approximations for the cross section. Compared to a direct mass determination from the reconstruction of the top-quark decay products, this method leads to larger uncertainties. However, the extracted mass corresponds to a theoretically well-defined renormalization scheme, e.g $\overline{\mathrm{MS}}$ or pole scheme. Here we show the effect of the inclusion of the exact NNLO result for the $q \bar{q}$ channel and of higher-order effects from NNLL resummation on the extraction of the pole mass $m_{t}$ from the Tevatron data.

The central value of the pole mass $m_{t}$ is given by the maximum of a likelihood function obtained from the convolution of two normalized gaussians centred at the theoretical and experimental values respectively, with widths given by the total theoretical error, obtained from the linear sum of theory and $\mathrm{PDF}+\alpha_{s}$ uncertainty, and experimental error. The mass dependence of the experimentallymeasured cross section is obtained from [3] and is plotted in Figure 3, alongside the mass dependence of our NNLL result.

Using our best NNLL result as theoretical input we extracted the pole mass $m_{t}=171.4_{-5.7}^{+5.4} \mathrm{GeV}$, in good agreement with the value from direct mass reconstruction 
$m_{t}=173.2 \pm 0.8 \mathrm{GeV}$. The value obtained using the approximated NNLO result as theory input is $m_{t}=171.0_{-6.3}^{+5.8}$, while the exact NNLO calculation gives $m_{t}=170.5_{-6.4}^{+5.3}$. This shows that the effect of the exact $O\left(\alpha_{s}^{4}\right)$ terms and higher-order contributions from resummation on the central value is moderate, while a reduction of the error is observed for the resummed result.

The CMS collaboration has recently published the most precise measurement of the $t \bar{t}$ cross section to date [1], from which, using our NNLL prediction, we obtained the pole mass $m_{t}=174.3_{-4.4}^{+4.9} \mathrm{GeV}$. This is in even better agreement with the direct-reconstruction value and has an error of less than $\pm 3 \%$. Note that all results shown are obtained assuming that the Monte Carlo mass parameter which enters the determination of the experimental cross section can be identified with the pole mass. Allowing for a difference of $\pm 1 \mathrm{GeV}$ between the two masses translates into an additional uncertainty of $\pm(0.4-0.5) \mathrm{GeV}$ on the extracted mass.

\section{Conclusions}

We have presented updated results for the total top-pair cross section at Tevatron and LHC which include simultaneous resummation of soft and Coulomb effects, boundstate contributions and the recent exact NNLO result for the $q \bar{q}$ channel. Our best predictions,

$$
\begin{array}{rll}
\text { Tevatron } & : & 7.15_{-0.20-0.25}^{+0.21+0.30} \mathrm{pb}, \\
\text { LHC }(\sqrt{s}=7 \mathrm{TeV}) & : & 162.4_{-6.9-6.8}^{+6.7+7.3} \mathrm{pb}, \\
\mathrm{LHC}(\sqrt{s}=8 \mathrm{TeV}) & : & 231.8_{-9.9-9.1}^{+9.6+9.8} \mathrm{pb},
\end{array}
$$

show a good agreement with experimental measurements, and display a residual theoretical uncertainty of $\pm(3-4) \%$ and an additional $\pm(4-4.5) \%$ error from the inputs for PDFs and $\alpha_{s}$. The dependence of the resummed result on different PDF sets was found to be small at Tevatron, though a larger discrepancy between different PDF parameterizations is observed at the LHC. Our NNLL prediction was used to extract the top-quark pole mass from Tevatron and LHC data, which resulted in values in good agreement with direct mass measurements and with a total error of the mass determination of $\pm 3 \%$ or better.

\section{References}

[1] S. Chatrchyan et al. [CMS Collaboration], JHEP 1211, 067 (2012) [arXiv:1208.2671 [hep-ex]].

[2] ATLAS Collaboration, conf. note ATLAS-CONF2012-024.

[3] V. M. Abazov et al. [D0 Collaboration], Phys. Lett. B 704, 403 (2011) [arXiv:1105.5384 [hep-ex]].

[4] CDF Collaboration, conf. note 9913, 2009.

[5] P. Nason, S. Dawson and R. K. Ellis, Nucl. Phys. B 303, 607 (1988).

[6] P. Bärnreuther, M. Czakon and A. Mitov, Phys. Rev. Lett. 109, 132001 (2012) [arXiv:1204.5201 [hep-ph]] and arXiv:1206.0621 [hep-ph].
[7] M. Czakon and A. Mitov, JHEP 1212, 054 (2012) [arXiv:1207.0236 [hep-ph]] and arXiv:1210.6832 [hepph].

[8] N. Kidonakis and G. F. Sterman, Nucl. Phys. B 505, 321 (1997) [hep-ph/9705234].

[9] R. Bonciani, S. Catani, M. L. Mangano and P. Nason, Nucl. Phys. B 529, 424 (1998) [Erratum-ibid. B 803, 234 (2008)] [hep-ph/9801375].

[10] T. Becher and M. Neubert, Phys. Rev. D 79, 125004 (2009) [Erratum-ibid. D 80, 109901 (2009)] [arXiv:0904.1021 [hep-ph]].

[11] A. Ferroglia, M. Neubert, B. D. Pecjak and L. L. Yang, Phys. Rev. Lett. 103, 201601 (2009) [arXiv:0907.4791 [hep-ph]].

[12] M. Beneke, P. Falgari and C. Schwinn, Nucl. Phys. B 828, 69 (2010) [arXiv:0907.1443 [hep-ph]].

[13] M. Czakon, A. Mitov and G. F. Sterman, Phys. Rev. D 80, 074017 (2009) [arXiv:0907.1790 [hep-ph]].

[14] N. Kidonakis, Phys. Rev. D 82, 114030 (2010) [arXiv:1009.4935 [hep-ph]].

[15] M. Aliev, H. Lacker, U. Langenfeld, S. Moch, P. Uwer and M. Wiedermann, Comput. Phys. Commun. 182, 1034 (2011) [arXiv:1007.1327 [hep-ph]].

[16] V. Ahrens, A. Ferroglia, M. Neubert, B. D. Pecjak and L. -L. Yang, JHEP 1109, 070 (2011) [arXiv:1103.0550 [hep-ph]].

[17] M. Cacciari, M. Czakon, M. Mangano, A. Mitov and P. Nason, Phys. Lett. B 710, 612 (2012) [arXiv:1111.5869 [hep-ph]].

[18] M. Beneke, P. Falgari and C. Schwinn, Nucl. Phys. B 842, 414 (2011) [arXiv:1007.5414 [hep-ph]].

[19] M. Beneke, P. Falgari, S. Klein and C. Schwinn, Nucl. Phys. B 855, 695 (2012) [arXiv:1109.1536 [hep$\mathrm{ph}]]$.

[20] M. Beneke, P. Falgari, S. Klein, J. Piclum, C. Schwinn, M. Ubiali and F. Yan, JHEP 1207, 194 (2012) [arXiv:1206.2454 [hep-ph]].

[21] M. Beneke, M. Czakon, P. Falgari, A. Mitov and C. Schwinn, Phys. Lett. B 690, 483 (2010) [arXiv:0911.5166 [hep-ph]].

[22] A. D. Martin, W. J. Stirling, R. S. Thorne and G. Watt, Eur. Phys. J. C 63, 189 (2009) [arXiv:0901.0002 [hep-ph]].

[23] H. -L. Lai, M. Guzzi, J. Huston, Z. Li, P. M. Nadolsky, J. Pumplin and C. -P. Yuan, Phys. Rev. D 82, 074024 (2010) [arXiv:1007.2241 [hep-ph]]; P. Nadolsky, J. Gao, M. Guzzi, J. Huston, H. -L. Lai, Z. Li, J. Pumplin and D. Stump et al., arXiv:1206.3321 [hepph].

[24] R. D. Ball, V. Bertone, F. Cerutti, L. Del Debbio, S. Forte, A. Guffanti, J. I. Latorre and J. Rojo et al., Nucl. Phys. B 849, 296 (2011) [arXiv:1101.1300 [hep$\mathrm{ph}]]$.

[25] S. Alekhin, J. Blümlein and S. Moch, Phys. Rev. D 86, 054009 (2012) [arXiv:1202.2281 [hep-ph]].

[26] CDF Collaboration, conf. note 10926, 2012; D0 Collaboration, D0 note 6363-CONF, 2012.

[27] CMS Collaboration, CMS PAS TOP-12-007, 2012. 
[28] M. Czakon and A. Mitov, arXiv:1112.5675 [hep-ph].
[29] V. M. Abazov et al. [D0 Collaboration], Phys. Lett. B 703, 422 (2011) [arXiv:1104.2887 [hep-ex]]. 